\title{
Energy distribution of electrons in an out-of-equilibrium metallic wire
}

\author{
H. Pothier, S. Guéron, Norman O. Birge ${ }^{\star}$, D. Esteve, M.H. Devoret \\ Service de Physique de 1'Etat Condensé, Commissariat à l'Energie Atomique, Saclay, F-91191 Gif-sur-Yvette Cedex, France
}

Z. Phys. B 103, 313-318 (1997)

Due to a technical error the name of the last author was not printed.

\begin{abstract}
We have measured with a tunnel probe the energy distribution function of quasiparticles in the middle of a $1.5 \mu \mathrm{m}$-long copper wire placed between two reservoirs at different potentials. We find a distribution function close to half the sum of the Fermi distributions in the reservoirs. The deviation from this behavior is not yet understood.
\end{abstract}

PACS: 71.10.Ay; 73.23.-b; 74.80.Fp

\section{Introduction}

The model of "non-interacting electrons" accounts for most electronic properties of bulk metals [1] and is justified by the Landau theory of Fermi Liquids [2]. This theory shows that the excitations of an electronic fluid are in fact not electrons, but "quasiparticles" which, to good approximation, are noninteracting. A quasiparticle can be viewed as an electron (or a hole) surrounded by a screening cloud. In a bulk metal, the residual interaction between quasiparticles is small because of efficient screening [2], and the picture of "non-interacting electrons" is justified. In diffusive mesoscopic conductors, however, screening is less efficient, and the validity of the Landau theory of Fermi liquids can be questioned. Theoretically, Altshuler et al. calculated that even in this case, the quasiparticle lifetime remains large [3], at least for dimensions larger than one.

We present here an experiment which directly probes quasiparticle energy relaxation in a mesoscopic wire placed in a stationary out-of-equilibrium situation. We have measured the distribution function of quasiparticles as a function of energy in the middle of a thin metallic wire of length $L=1.5 \mu \mathrm{m}$ connected at its ends to two thick and large electrodes at different potentials (see Fig. 1). These electrodes act as electron reservoirs in the sense of Landauer [4]: they absorb all incoming quasiparticles, and emit quasiparticles with an energy distribution given by their own Fermi distribution. As a consequence, the typical residence time of quasiparticles in the wire is the diffusion time $\tau_{D}=L^{2} / D$, USA.

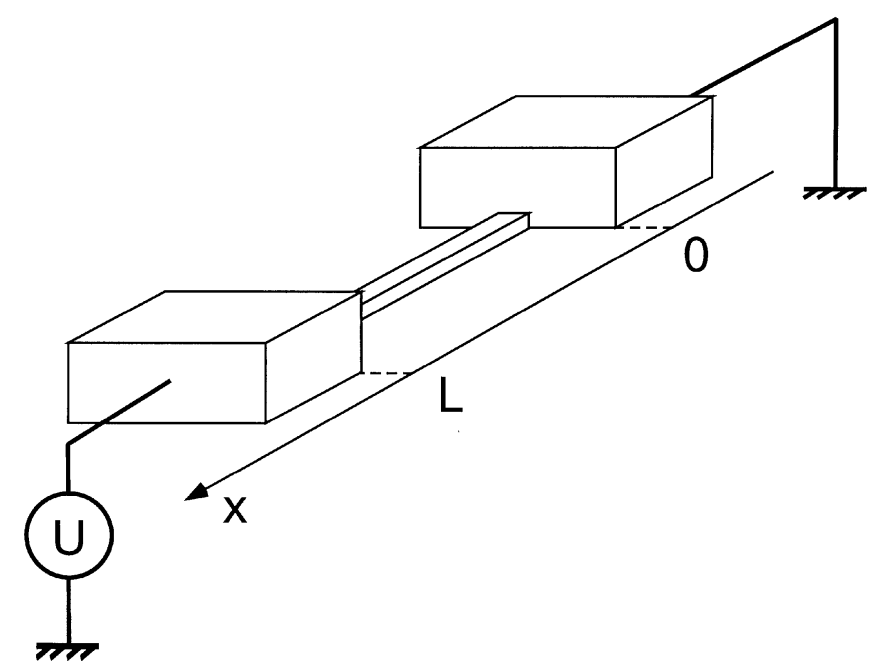

Fig. 1. Experimental layout: a wire of length $L$ is placed between two large and thick electrodes. A voltage $U$ is applied between the pads

where $D$ is the diffusion constant of the electrons. The distribution function $f(x, E)$ at a distance $x$ from the right end of the wire and at an energy $E$ reflects how much quasiparticles loose energy during the diffusion time. In particular, if $\tau_{D}$ is short compared with the typical interaction time, each quasiparticle travels through the wire at constant energy, and $f(x, E)$ is a linear combination of the distribution functions in the reservoirs, weighted by the proportion of quasiparticles coming from each reservoir at that point.

In Sect. 2, we discuss the quasiparticle energy distribution function in a wire in three limiting regimes: non interacting quasiparticles, strong phonon scattering and strong quasiparticle scattering without the presence of phonon scattering. In Sect. 3, we describe the measurement of the distribution function, which is performed using a tunnel junction between the wire and a superconducting electrode. In Sect. 4, the experimental setup is presented. Section 5 we devote to the experimental results: we have measured the distribution function of quasiparticles in a $1.5 \mu \mathrm{m}$-long wire for several values of the voltage across the wire. Finally, in Sect. 6, we review the predictions derived by Blanter [5] along the lines of Altshuler et al. [3], and compare them with our experimental results. 


\section{Energy distribution function in a wire placed between reservoirs at different electrical potentials}

We choose as the reference energy the electrochemical potential of the reservoir situated at $x<0$. When the reservoir at $x>L$ is biased at potential $U$, its electrochemical potential is $-e U$. The distribution functions in the reservoirs are therefore Fermi-Dirac functions at the temperature $T$ of the reservoirs, shifted in energy by $e U$. The shape of the distribution function $f(x, E)$ in the wire is determined by balancing the inelastic scattering processes and the diffusion. We consider here the quasiparticle scattering mechanisms expected in metals at energies of the order of $1 \mathrm{~K}$ : scattering by phonons and scattering by quasiparticles. Several authors have evaluated the distribution function of quasiparticles for this system in several limits in order to calculate the shot noise $[6,7,8]$. We now recall their results in three limiting regimes.

\subsection{No quasiparticle scattering, no phonon scattering}

In the absence of inelastic scattering, the total energy of each quasiparticle is conserved along the wire. The distribution function $f(x, E)$ reflects the probability to find an electron at position $x$ and energy $E$, and obeys the quasiclassical Boltzmann equation [6]

$$
\frac{\partial f(x, E)}{\partial t}-D \frac{\partial^{2} f(x, E)}{\partial x^{2}}=0
$$

The stationary solution of this equation, given the boundary conditions at the ends of the wire, is:

$f(x, E)=\left(1-\frac{x}{L}\right) \mathbf{f}_{\mathbf{T}}(\mathbf{E})+\frac{\mathbf{x}}{\mathbf{L}} \mathbf{f}_{\mathbf{T}}(\mathbf{E}+\mathbf{e U})$

where $\mathbf{f}_{\mathbf{T}}(\mathbf{E})=\mathbf{1} /\left(\mathbf{1}+\exp \left(\mathbf{E} / \mathbf{k}_{\mathbf{B}} \mathbf{T}\right)\right)$ is the Fermi-Dirac function at temperature $T$. In this case, neither the electrochemical potential, nor the electronic temperature is defined. If $k_{B} T \ll e U$, the distribution function has a step at $f(x, E)={ }_{L}^{x}$ for $-e U<E<0$. A plot of the distribution function given by Eq. (2) as a function of position is shown in Fig. 2.

\subsection{Strong quasiparticle scattering, no phonon scattering}

In the case of strong quasiparticle scattering and in absence of phonon scattering, thermal equilibrium is achieved locally. The distribution function is a Fermi-Dirac function: $f(x, E)=\mathbf{f}_{\mathbf{T}_{\mathbf{e}}(\mathbf{x})}(\mathbf{E}-\mu(\mathbf{x}))$ where $\mu(x)=-e U_{L}^{x}$ is the local electrochemical potential, and $T_{e}(x)$ the local electron temperature. The temperature $T_{e}(x)$ obeys the heat equation $[9,10,8]$ :

$$
\frac{\partial}{\partial x}\left(\kappa \frac{\partial T_{e}}{\partial x}\right)+\frac{1}{S L} \frac{U^{2}}{R}=0 .
$$

where $\kappa$ is the electronic heat conductivity and $S$ the crosssectional area of the wire. Using the Wiedemann-Franz law $\kappa=\mathscr{C} \sigma T_{e}$, where $\mathscr{B}=\frac{\pi^{2}}{3}\left(\begin{array}{c}k_{B} \\ e\end{array}\right)^{2}$ is the Lorenz number, and given the boundary conditions $T_{e}(0)=T_{e}(L)=T$ we find

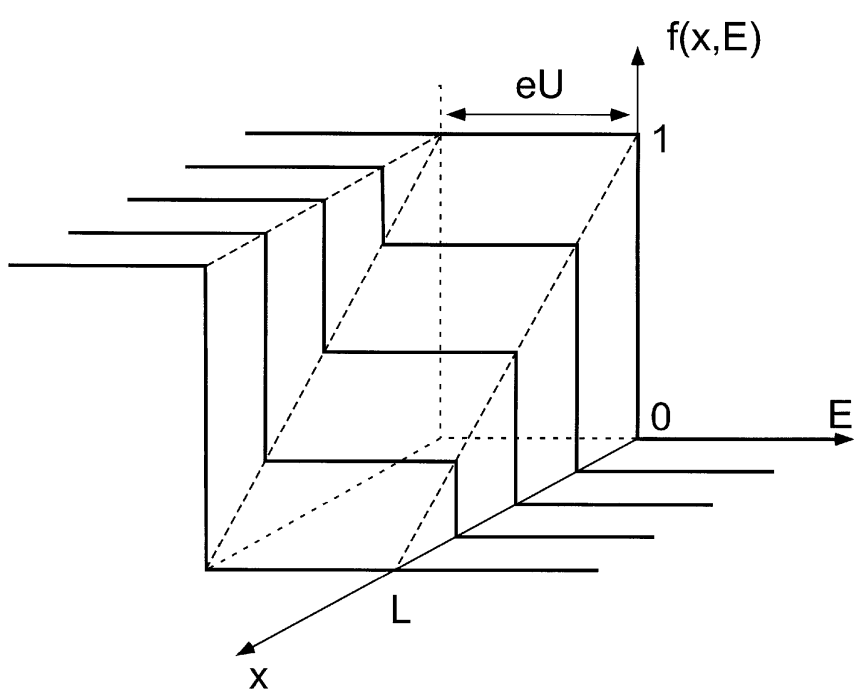

Fig. 2. Distribution function $f(x, E)$ as a function of energy $E$ (horizontal axis) and position $x$ (oblique axis) in the non-interacting quasiparticle limit

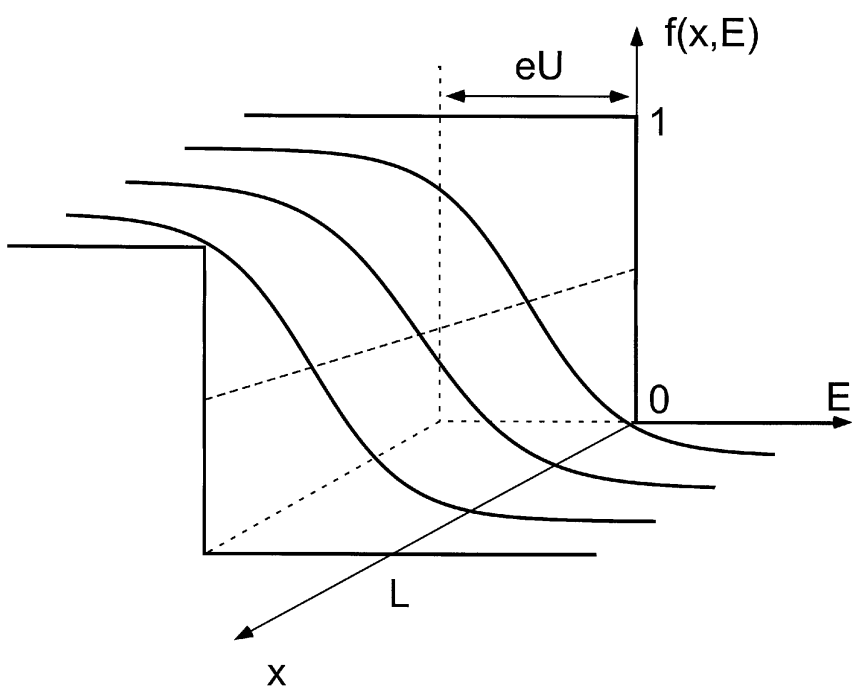

Fig. 3. Distribution function $f(x, E)$ as a function of energy $E$ (horizontal axis) and position $x$ (oblique axis) in the limit where phonon scattering is negligible, but quasiparticle scattering is strong

$T_{e}(x)=\sqrt{T^{2}+{ }_{L}^{x}\left(1-{ }_{L}^{x}\right)} \frac{1}{\mathscr{C}} U^{2}$. The space dependence of the resulting distribution function is shown in Fig. 3 for $T=0$. The characteristic shot noise in this "hot electron regime" has recently been measured by Steinbach et al. [10].

\subsection{Strong phonon scattering limit}

In presence of strong phonon scattering, electrons thermalize with the phonons and $f(x, E)=\mathbf{f}_{\mathbf{T}}(\mathbf{E}-\mu(\mathbf{x}))$ with $\mu(x)=$ $-e U_{L}^{x}$. In Fig. 4, we show the space dependence of the distribution function.

\section{Measurement of the distribution function}

We take advantage of the known anomalous density of states of a superconducting probe electrode to sense the distribution function in the middle of the wire through a tunnel 


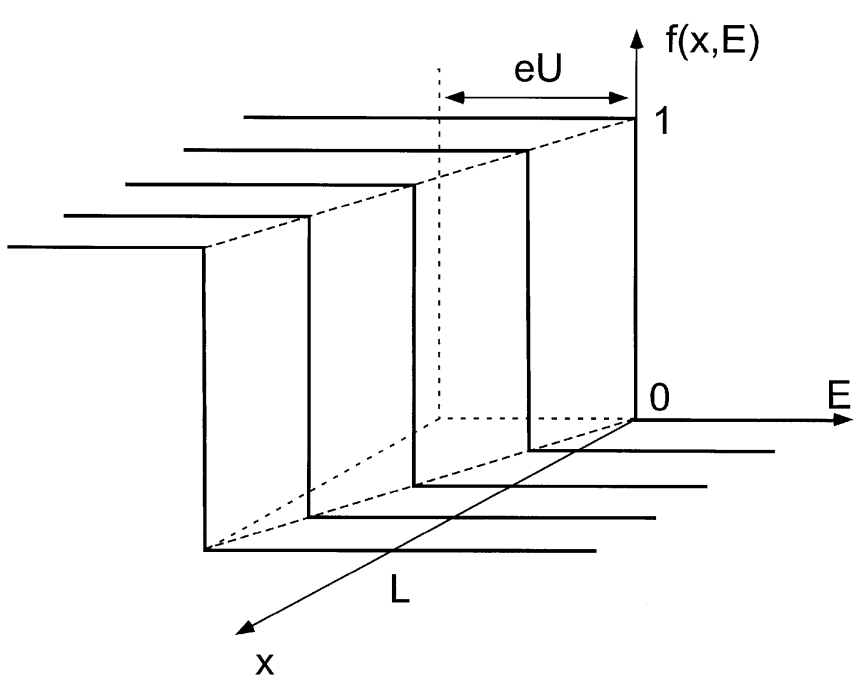

Fig. 4. Distribution function $f(x, E)$ as a function of energy $E$ (horizontal axis) and position $x$ (oblique axis) in the limit of strong phonon scattering

junction. More precisely, we place an aluminum superconducting electrode oxidized on its surface below the central part of the wire. We measure the differential conductance $\frac{d I}{d V}$ as a function of voltage $V$ of this opaque tunnel junction . Disregarding charging effects [12] the current through the junction is given by

$$
\begin{aligned}
I(V)= & \frac{1}{e R_{T}} \int_{-\infty}^{+\infty} d E n_{S}(E-e V) \\
& \times\left\{f_{S}(E-e V)\left(1-f_{N}(E)\right)\right. \\
& \left.-f_{N}(E)\left(1-f_{S}(E-e V)\right)\right\},
\end{aligned}
$$

where $R_{T}$ is the tunnel junction resistance, $n_{S}(E)=E$ / $\sqrt{ } E^{2}-\Delta^{2}$ is the spectrum of the superconductor [13], $\Delta$ being the gap energy of the superconductor, $f_{N}(E) \equiv$ $f\left(\frac{L}{2}, E\right)$ is the distribution function in the wire at the position of the tunnel junction, and $f_{S}(E)$ is the distribution function in the superconductor. When $k_{B} T \ll \Delta$, $n_{S}(E) f_{S}(E) \simeq n_{S}(E)(1-\Theta(E))$ where $\Theta(E)$ is the Heavyside function, and we find:

$$
\begin{aligned}
\frac{d I}{d V}(V)= & \frac{1}{R_{T}} \int_{-\infty}^{+\infty} d E \frac{\partial n_{S}}{\partial E}(E-e V) \\
& \times\left\{f_{N}(E)+\Theta(E-e V)-1\right\} .
\end{aligned}
$$

\section{Experimental setup and sample fabrication}

The experiment was performed at sub-Kelvin temperature and with voltages $U$ below $1 \mathrm{mV}$ in order to avoid phonon scattering [2]. We chose $L=1.5 \mu \mathrm{m}$, a length at which the non-interacting quasiparticle regime was almost reached in shot noise experiments [10]. The good contact between the reservoirs and the wire was obtained by evaporating the entire device in a single pump down, through a suspended mask.

The mask was fabricated by electron beam lithography on a trilayer of MAA, germanium and PMMA deposited on an oxidized silicon wafer. After exposure of the PMMA and development, the pattern was transferred to the Ge layer by reactive ion etching (RIE). The MAA layer was etched out

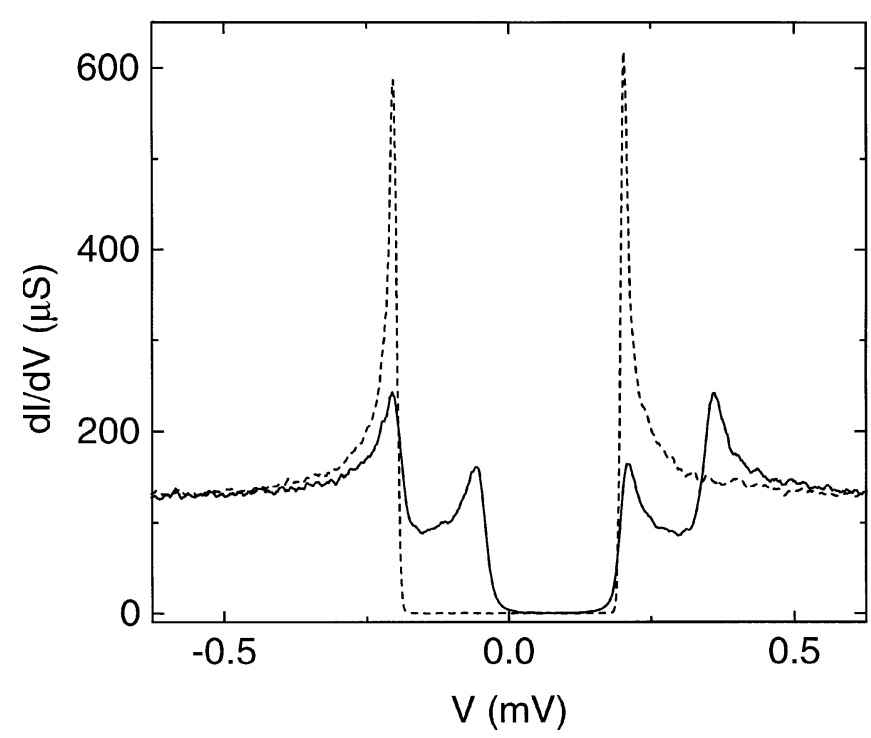

Fig. 5. Differential conductivity $d I / d V$ of the tunnel junction placed at the middle of the wire, as a function of the voltage $V$ for two values of the voltage $U$ across the wire: $U=0$ (dashed line) and $U=0.15 \mathrm{mV}$ (solid line)

through the openings of the Ge mask first by RIE, then in a MIBK:propanol solution. In a vacuum, we first evaporated $30 \mathrm{~nm}$ of aluminum at an angle so as to produce the superconducting probe electrode. The parasitic aluminum image of the wire landed on the edge of the MAA layer sustaining the Ge mask, and was lifted-off along with the MAA at the end of the whole process. The surface of aluminum was oxidized in an $\mathrm{Ar} / \mathrm{O}_{2}$ mixture. Perpendicular to the substrate, we then evaporated $45 \mathrm{~nm}$ of copper in order to produce the wire and the bottom part of the large pads. The tunnel junction was obtained at the $300 \times 110 \mathrm{~nm}^{2}$ overlap of the two evaporated films in the middle of the wire. During the two first evaporations, the slit in the mask defining the wire was filled, and the subsequent evaporation of $500 \mathrm{~nm}$ of copper at a large angle only thickened the large pads. After lift-off in acetone, we thus obtained a single image of the wire, which overlaps the superconductor in the middle, forming a junction, and which opens at its ends into millimetric size, thick pads.

\section{Distribution function at the middle of a $1.5 \mu \mathrm{m}$-long wire}

The sample was thermally anchored to the mixing chamber of a dilution refrigerator, and cooled down to $20 \mathrm{mK}$. Voltages were applied and measured through properly filtered coaxial lines [14]. From the resistance of the wire at $20 \mathrm{mK}$, $R=14.5 \Omega$, from the nominal thickness of the copper film and from the width of the wire measured on a SEM picture, we deduce the diffusion constant $D=65 \mathrm{~cm}^{2} / \mathrm{s}$, and the diffusion time $\tau_{D}=350 \mathrm{ps}$.

In Fig. 5, we show the ${ }_{d V}^{d I}(V)$ characteristic of the tunnel junction for both $U=0$ (dashed curve) and $U=0.15 \mathrm{mV}$ (solid curve). Fitting the curve for $U=0$ to expression (5) with $f_{N}$ being a Fermi function at a fit temperature $T_{e}$, we found $T_{e}=30 \mathrm{mK}$, in satisfactory agreement with the measured temperature, the energy gap of the aluminum elec- 




Fig. 6. Distribution function $f_{N}(E)$ at $x=L / 2$, obtained from the deconvolution of the curves of Fig. 5 using (5). We have added dotted lines at $E=0$ and $E=-0.15 \mathrm{meV}$

trode $\Delta=0.2 \mathrm{meV}$, and the tunnel resistance of the junction $R_{T}=8 \mathrm{k} \Omega$. We used the values of $\Delta$ and $R_{T}$ to deconvolve the $\frac{d I}{d V}(V)$ characteristics via expression (5) and thus determined $f_{N}(E)$. From the characteristics of Fig. 5, we obtain the distribution function shown in Fig. 6. The shape of the distribution function when $U=0$ (dashed curve) fits perfectly to a Fermi-Dirac function at $T_{e}=30 \mathrm{mK}$ (comparison not shown). The distribution function deduced from the curve at $U=0.15 \mathrm{mV}$, in contrast, does not resemble a Fermi-Dirac function: it flattens out around the value 0.5, and displays maximal slopes at $E=0$ and $E=-0.15 \mathrm{meV}$ (see vertical dotted lines). In this respect, the function resembles the non-interacting quasiparticle case (Fig. 2), in which the slope of the distribution function is maximal at $E=0$ and $E=-e U$. That the step is not exactly positioned at 0.5 but a little bit below can be explained by a shift of $20 \mathrm{~nm}$ of the position of the junction from the center of the wire. However, the steepness of the curve at $E=0$ and $E=-0.15 \mathrm{meV}$ is less than half the one of the Fermi distributions in the reservoirs, as was expected from Eq. (2). Moreover, the curve is not flat near the value 0.5. When allowing for $T$ to be larger than $30 \mathrm{mK}$ in Eq. (2), we could reproduce the rounding of the steps, but not its slope.

In Fig. 7, we show a series of distribution functions obtained from the measurements at $U=0$ to $U=0.3 \mathrm{mV}$ by steps of $0.05 \mathrm{mV}$. The width of the step near 0.5 is well given by $e U$, as expected, whereas the rounding increases and the slope of the step decreases when $U$ is increased. Note that in a clean metal, where the energy relaxation rate due to quasiparticle interactions is proportional to the square of the energy, one would expect the distribution function to tend rapidly towards a Fermi distribution when $U$ is increased.

\section{Theoretical predictions for quasiparticle interactions in a diffusive metal}

As we have shown in Sect. 5, the experimental distribution function resembles the one expected from the "independentelectron" picture, but is more rounded. The way quasiparticle interactions redistribute the energy among the quasiparticles

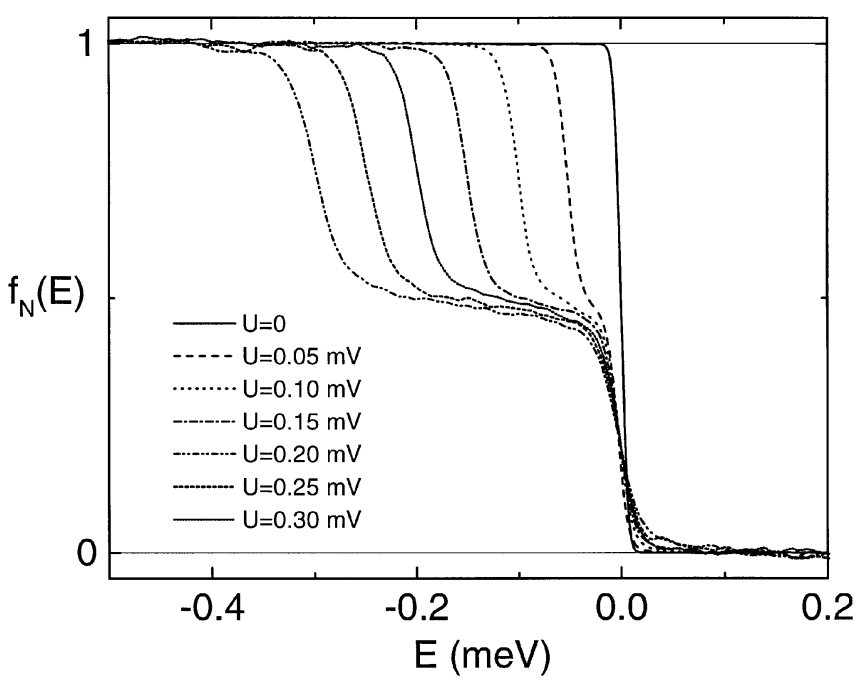

Fig. 7. Distribution function $f_{N}(E)$ at $x=L / 2$, obtained from the measurement of the $d I / d V-V$ characteristics of the tunnel junction for $U$ varying from 0 to $0.3 \mathrm{mV}$ by steps of $0.05 \mathrm{mV}$

has been recently calculated by Blanter for our experimental situation [5], along the lines of Altshuler et al. [3]. We present his derivation, and a comparison with the data.

Electron-electron interactions come into a collision term $\Im(x, E)$ in the Boltzmann equation (1):

$\frac{\partial f(x, E)}{\partial t}-D \frac{\partial^{2} f(x, E)}{\partial x^{2}}=\Im(x, E)$

We limit ourselves to the simplest diagram in which two quasiparticles of energies $E$ and $E^{\prime}$ exchange an energy $\varepsilon$. The collision term is a sum of two terms, one decreasing the occupation factor at energy $E$ and the other one increasing it:

$\Im(x, E)=\Im^{\text {out }}(x, E)+\Im^{\text {in }}(x, E)$.

Each term contains a kernel function $K(\varepsilon)$ and four occupation factors:

$$
\begin{aligned}
\Im^{\text {out }}= & -\int \mathrm{d} \varepsilon \int \mathrm{d} E^{\prime} K(\varepsilon) \\
& \times f(x, E) f\left(x, E^{\prime}\right)(1-f(x, E-\varepsilon)) \\
& \left(1-f\left(x, E^{\prime}+\varepsilon\right)\right) ; \\
\Im^{\text {in }}= & \int \mathrm{d} \varepsilon \int \mathrm{d} E^{\prime} K(\varepsilon) \\
& \times f(x, E-\varepsilon) f\left(x, E^{\prime}+\varepsilon\right)(1-f(x, E)) \\
& \left(1-f\left(x, E^{\prime}\right)\right) .
\end{aligned}
$$

Assuming that the interaction is local on the scale of the distribution function variation, we have taken all occupation factors at the same position $x$. The kernel $K(\varepsilon)$ accounts for the diffusive motion of the interacting quasiparticles, on the one hand, and for their Coulomb interaction screened by the diffusive electronic fluid, on the other hand:

$$
K(\varepsilon) \propto \frac{\pi}{L} \sum_{q L / \pi=1}^{\infty}|V(q, \varepsilon)|^{2}\left\{\operatorname{Re}\left(\frac{1}{D q^{2}-i \varepsilon}\right)\right\}^{2} .
$$

Since in our wire of finite length the diffusive motion decomposes on discrete modes, a sum replaces here the integral 
on $q$ in Altshuler et al. [3]. The mode $q=0$ was discarded because the system is open. Moreover, we have only kept the decomposition on the longitudinal modes since the times corresponding to the energies addressed in our experiment (up to $0.3 \mathrm{meV}$ ) are larger than the diffusion time in the transversal dimensions. The screened Coulomb interaction is expressed in $q$ space as a function of the polarizability $\Pi(q, \varepsilon)$ of the electron gas:

$V(q, \varepsilon)=\frac{V_{0}(q)}{1+\Pi(q, \varepsilon) V_{0}(q)}$

where $V_{0}(q)$ is the bare Coulomb potential. Under the assumption that screening is due to the diffusive motion of the quasiparticles themselves, one takes

$\Pi(q, \varepsilon)=\nu S \frac{D q^{2}}{D q^{2}-i \varepsilon}$

where $\nu$ is the density of states of the metal and $S$ is the cross-section of the wire. In a metal, $\nu$ is so large that Eq. (11) simplifies to

$V(q, \varepsilon)=\frac{1}{\Pi(q, \varepsilon)}$.

One obtains then:

$K(\varepsilon)=K_{0} g\left(\sqrt{ } 2 \varepsilon / E_{C}\right)$

where $K_{0}=8 /\left(\pi \hbar \nu S L E_{C}^{2}\right), E_{C}=\hbar / \tau_{D}$ and

$g(u)=-\frac{4}{u^{4}}+\frac{2 \sinh u+\sin u}{u^{3} \cosh u-\cos u}$.

Asymptotically, for large values of $\varepsilon, K(\varepsilon)=\frac{K_{0}}{\sqrt{ } 2}\left(\begin{array}{c}E_{C} \\ \varepsilon\end{array}\right)^{3 / 2}$.

We have numerically calculated the steady state solution of the Boltzmann equation (6) using this prediction, and with a reservoir temperature of $30 \mathrm{mK}$. When we take the theoretical value $K_{0}=7 \mathrm{ps}^{-1} \mathrm{meV}^{-2}$, we find no visible effect of electron-electron interactions on the distribution function. Allowing for $K_{0}$ to be a fit parameter, we found the best fit for the curve at $U=0.3 \mathrm{meV}$ with $K_{0}=100 \mathrm{ps}^{-1} \mathrm{meV}^{-2}$. The corresponding curve, and those obtained with the same value of $K_{0}$ at $U=0.1 \mathrm{meV}$ and $U=0.2 \mathrm{meV}$, are compared in Fig. 8 to the experimental data. The calculation produces a too small slope for $U=0.1 \mathrm{meV}$ and $U=0.2 \mathrm{meV}$. Moreover, the rounding at $E=0$ and $E=-e U$ is not obtained in any case.

\section{Conclusions}

We have measured in a direct and novel way the distribution function of quasiparticles in the middle of a wire in a nonequilibrium situation: the two ends of the wire are biased at different potentials, and the distribution functions at the two ends of the wire are well imposed by the careful implementation of Landauer's concept of "reservoirs". We find that the shape of the distribution function is reminiscent of the non-interacting quasiparticle situation: it resembles half the sum of the two Fermi functions in the reservoirs shifted by the electrical potential. We find that the discrepancy from this ideal situation is not accounted for by present form of the existing theory of quasiparticle interactions in disordered

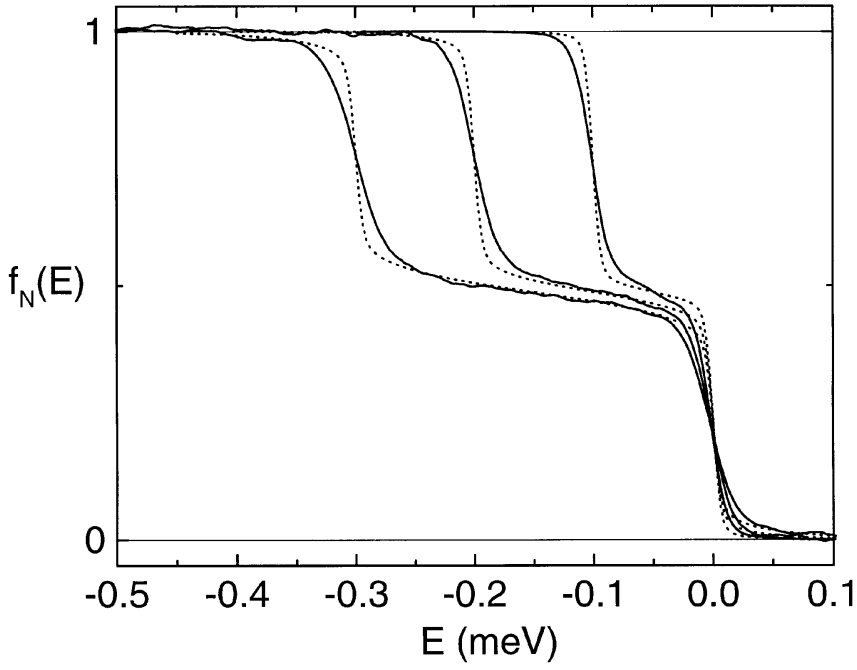

Fig. 8. Comparison between the experimental data (solid lines) at $U=$ $0.1 \mathrm{mV}, U=0.2 \mathrm{mV}$ and $U=0.3 \mathrm{mV}$, and the theoretical prediction (dotted lines) with $K_{0}=100 \mathrm{ps}^{-1} \mathrm{meV}^{-2}$

metals. Experiments on wires with different lengths are in progress in order to probe the balance between interactions and diffusion in different situations, and to experimentally access the energy dependence of the interaction.

We are grateful to Ya. Blanter for several discussions, and for communication of the results of his calculations. We thank L. Calvet for correcting the manuscript. N.O. B. acknowledges support of the NSF under grant DMR-9321850.

\section{References}

1. N.W. Ashcroft, N.D. Mermin, Solid State Physics, CBS Publishing (1975)

2. D. Pines, P. Nozière, The Theory of Quantum Liquids. 1: Normal Fermi Liquids, W.A. Benjamin (1966)

3. B.L. Altshuler, A.G. Aronov, in Electron-Electron Interactions in Disordered Systems, Ed. A.L. Efros, M. Pollak, Elsevier Science Publishers B.V. (1985)

4. R. Landauer, IBM J. Res. Develop. 1, 223 (1957); 32, 306 (1988)

5. Ya. Blanter, Phys. Rev. B 54, 12807 (1996) and private communications

6. K.E. Nagaev, Phys. Lett. A 169, 103 (1992); Phys. Rev. B 52, 4740 (1995)

7. V.I. Kozub, A. M. Rudin, Phys. Rev. B 52, 7853 (1995)

8. M.J.M. de Jong, C.W.J. Beenakker, Physica A 230, 219 (1996)

9. D.E. Prober, M.N. Wybourne, M. Kansakar, Phys. Rev. Lett. 75, 3964 (1995)

10. A.H. Steinbach, J.M. Martinis, M.H. Devoret, Phys. Rev. Lett. 76, 3806 (1996)

11. J. M. Rowell, in Tunneling Phenomena in Solids, edited by E. Burstein, S. Lundqvist (Plenum, New York, 1969), p. 385; T. Claeson, ibid., p. 443

12. M. H. Devoret, D. Esteve, H. Grabert, G.-L. Ingold, H. Pothier, C. Urbina, Phys. Rev. Lett. 64, 1824 (1990); G.-L. Ingold, Yu. V. Nazarov, in Single Charge Tunneling, edited by H. Grabert, M.H. Devoret (Plenum Press, New York, 1992), p. 21

13. M. Tinkham, Introduction to Superconductivity (Mc Graw Hill, 1985), p. 41

14. D. Vion, P.F. Orfila, P. Joyez, D. Esteve, M. H. Devoret, J. Appl. Phys. 77, 2519 (1995) 